\title{
Transapical transcatheter aortic valve implantation followed by transfemoral transcatheter edge-to-edge repair of the tricuspid valve using the MitraClip system \\ - a new treatment concept for an inoperable patient with significant aortic stenosis and severe tricuspid valve regurgitation
}

\author{
Marek Kowalski ${ }^{1}$, Norbert Franz ${ }^{1,2}$, Steffen Hofmann ${ }^{3}$, Frank Ritter ${ }^{1}$, Joachim Thale ${ }^{1}$, Henning Warnecke ${ }^{2,3}$ \\ ${ }^{1}$ Cardiology Department, Schuechtermann-Klinik, Bad Rothenfelde, Germany \\ ¿University Witten-Herdecke, Germany \\ ${ }^{3}$ Cardiac Surgery Department, Schuechtermann-Klinik, Bad Rothenfelde, Germany
}

Adv Interv Cardiol 2016; 12, 3 (45): 262-266

DOI: 10.5114 /aic.2016.61650

Transcatheter mitral valve edge-to-edge repair using the MitraClip system (Abbott Vascular, USA) and transcatheter aortic valve implantation (TAVI) are well established in high-risk or inoperable patients with severe mitral regurgitation and severe aortic stenosis. The benefits of both methods have been confirmed in large cohort studies [1-4]. Recently, successful transcatheter tricuspid valve edge-to-edge repair using the MitraClip system was described for high surgical risk or inoperable patients [5-7].

Here we present to our knowledge the first case of transapical TAVI (TA-TAVI) followed by transfemoral clipping of the tricuspid valve using the MitraClip system.

An 81-year-old male patient with a history of biventricular heart decompensation suffered from severe dyspnea on admission (New York Heart Association functional class III), lower leg edema, ascites, pleural effusions and stage III chronic kidney disease. Echocardiography showed significant low-flow/low-gradient aortic stenosis (mean gradient $20 \mathrm{~mm} \mathrm{Hg}$, aortic valve area $0.9 \mathrm{~cm}^{2}$ ). In addition, severe tricuspid valve regurgitation (TR) with tethered leaflets was found (vena contracta area $1.22 \mathrm{~cm}^{2}-3 D$ Echo, vena contracta width $10 \mathrm{~mm}$ ). The mitral valve showed only mild regurgitation. Left and right ventricular function values were reduced (ejection fraction both $35 \%$ - Simpson method). Systolic pulmonary artery pressure was slightly elevated (42 $\mathrm{mm} \mathrm{Hg}$ ). As a result of heart team discussion, the patient was de- clared as surgically inoperable and qualified for consecutive transapical transcatheter aortic valve implantation - not suitable for a transfemoral approach - followed by transfemoral edge-to-edge repair of the tricuspid valve using the MitraClip system.

Both procedures were performed under general anesthesia using two- and three-dimensional transesophageal echocardiography (TEE) (iE 33, Philips Healthcare, Netherlands) and fluoroscopy guidance (Axiom Artis Zeefloor AXH 1604, Siemens, Germany). Unfractionated heparin was administered aiming at an ACT of 250-300 s throughout the procedure.

For the transapical TAVI (on October, 19 $\left.{ }^{\text {th }}, 2015\right)$, the left ventricular apex was surgically exposed (small anterolateral minithoracotomy), an epicardial pacemaker probe (TME T bipolar, Osypka AG, Germany) was positioned, and the apical suture was prepared. The apex was then punctured and after balloon valvuloplasty (balloon catheter VACS II $28 \times 40$ mm, Osypka AG, Germany) (Figure 1) the Symetis introducer system with a crimped 27-mm valve (ACURATE TA Aortic Bioprosthesis SYM-SV27-001, Symetis SA, Switzerland) was positioned in the aortic valve annulus (Figure 2). After repeated angiography through the pigtail catheter, the valve was implanted completely; step one: release the "stabilization arches" and "upper crown" by rotating the delivery knob, step two: slightly pull on the delivery system and contact the "upper crown" with the

\section{Corresponding author:}

Marek Kowalski MD, PhD, Cardiology Department, Schuechtermann-Klinik, Ulmenallee 5-11, 49214 Bad Rothenfelde, Germany, phone: +49 542464130023, e-mail: MKowalski@schuechtermann-klinik.de

Received: 3.05.2016, accepted: 11.06.2016. 


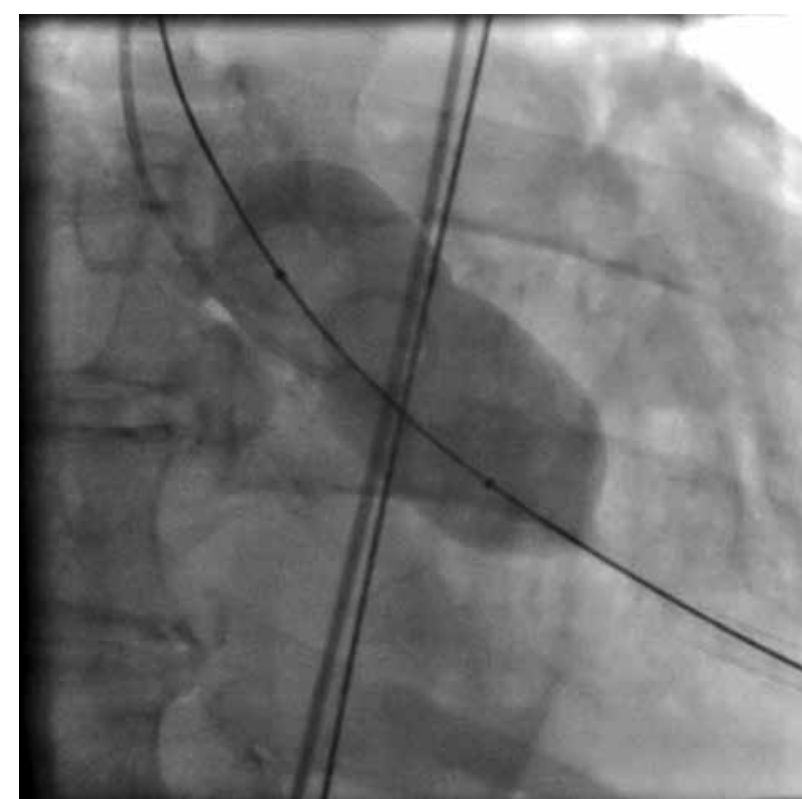

Figure 1. X-ray $\left(\mathrm{RAO} 0^{\circ}\right.$, cranial $\left.5^{\circ}\right)$ : aortic valvuloplasty under rapid pacing prior to TA-TAVI. Pigtail catheter positioned in aortic root

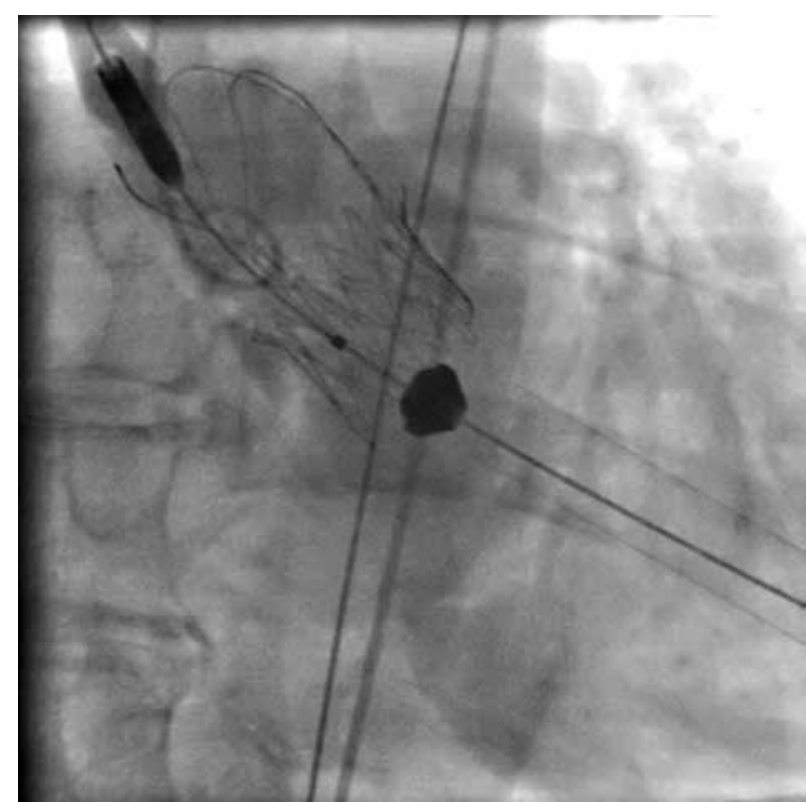

Figure 3. X-ray $\left(\mathrm{RAO} 0^{\circ}\right.$, cranial $\left.5^{\circ}\right)$ : acurate TA Aortic Bioprosthesis immediately after full deployment, delivery system not yet retracted. Pigtail catheter positioned in aortic root

leaflets, step three: the safety button is removed and the valve is fully released by rotating the delivery knob under rapid pacing (Figure 3). Final aortic angiography was performed, and the apex was closed after removing the introducer system. The procedure resulted in cor-

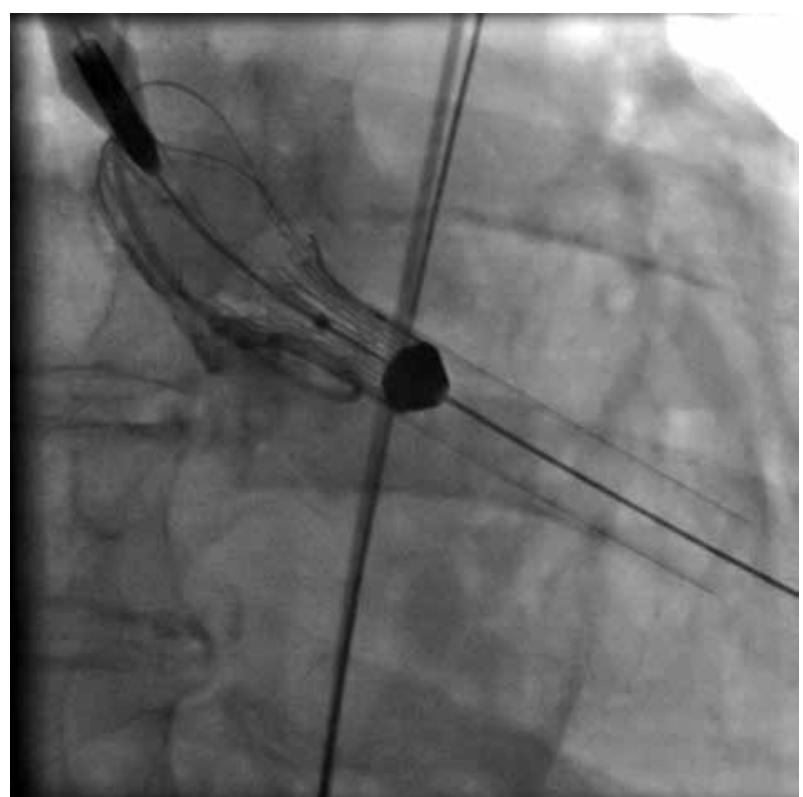

Figure 2. X-ray $\left(\mathrm{RAO} 0^{\circ}\right.$, cranial $\left.5^{\circ}\right)$ : acurate TA Aortic Bioprosthesis with delivery system in aortic annulus prior to implantation. The "stabilization arches" and "upper crown" at the distal part of the prosthesis are already unsheathed and partially released. Pigtail catheter positioned in aortic root

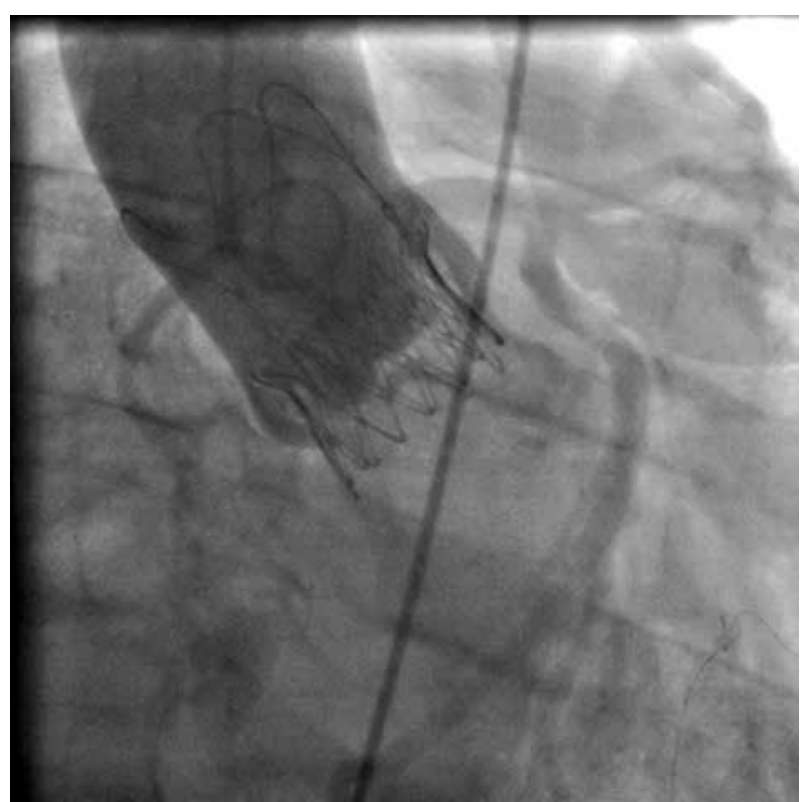

Figure 4. X-ray $\left(\mathrm{RAO} 0^{\circ}\right.$, caudal $\left.15^{\circ}\right)$ : final aortic angiography - acurate TA Aortic Bioprosthesis in correct position, aortic regurgitation trace. Pigtail catheter positioned in aortic root

rect positioning of the ACURATE TA Aortic Bioprosthesis with minimal aortic valve insufficiency (trace) (Figure 4). Echocardiography showed a mean gradient of $8 \mathrm{~mm} \mathrm{Hg}$ and an aortic valve area of $2.3 \mathrm{~cm}^{2}$. The patient left the hybrid operating room in a stable hemodynamic condi- 


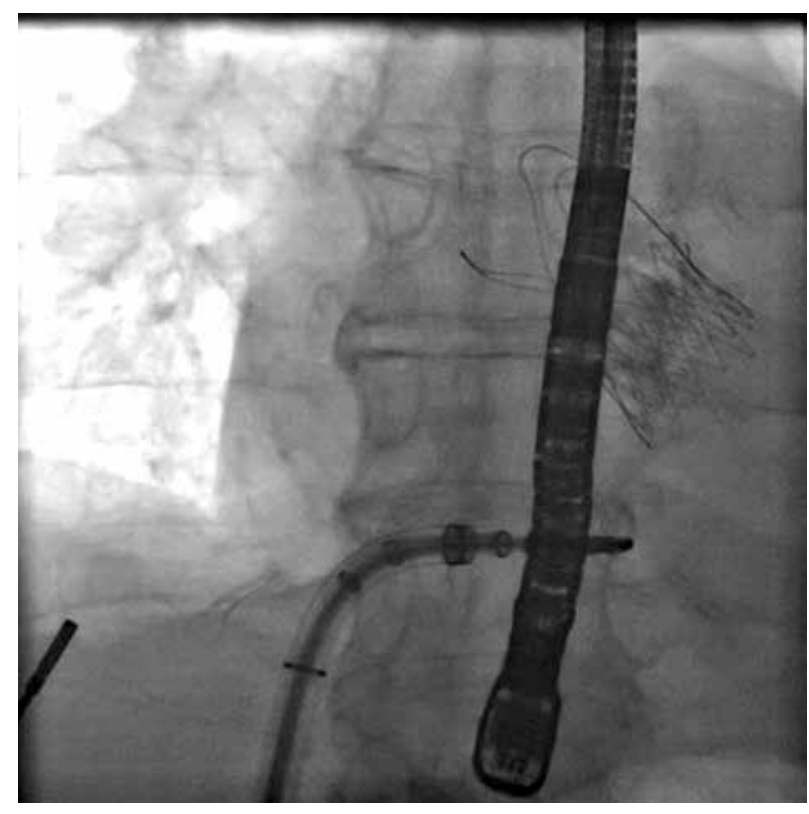

Figure 5. X-ray $\left(\mathrm{RAO} 0^{\circ}\right)$ : prior to tricuspid valve repair using the MitraClip system - steerable guide and clip delivery system placed in right atrium, clip arms closed. TEE probe in transesophageal position. Acurate TA Aortic Bioprosthesis implanted

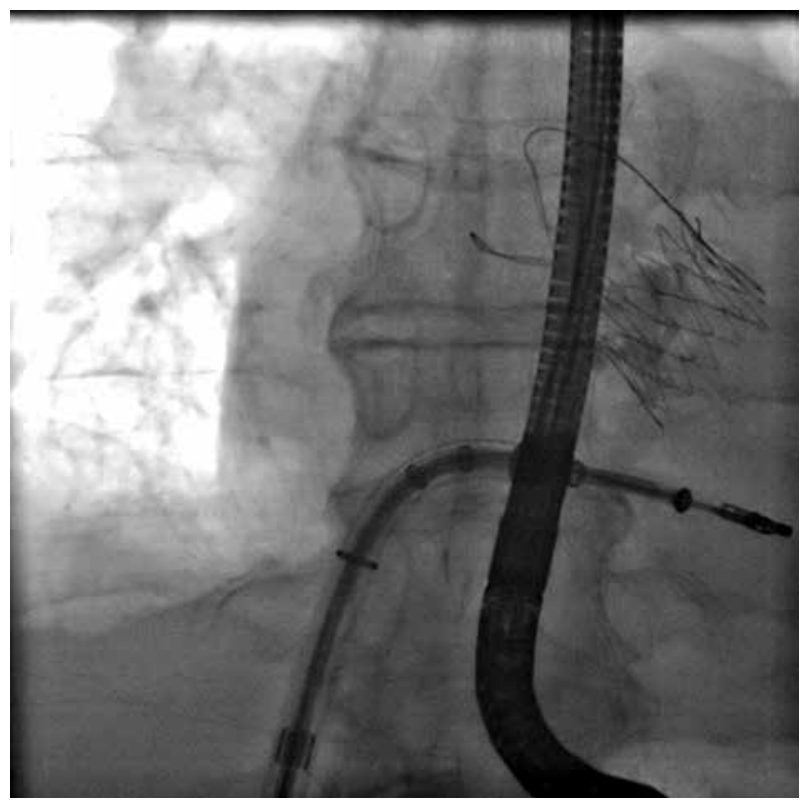

Figure 6. X-ray $\left(\mathrm{RAO} 0^{\circ}\right)$ : prior to tricuspid valve repair using the MitraClip system - steerable guide and clip delivery system placed in right ventricle, clip arms open. TEE probe in transgastric position. Acurate TA Aortic Bioprosthesis implanted
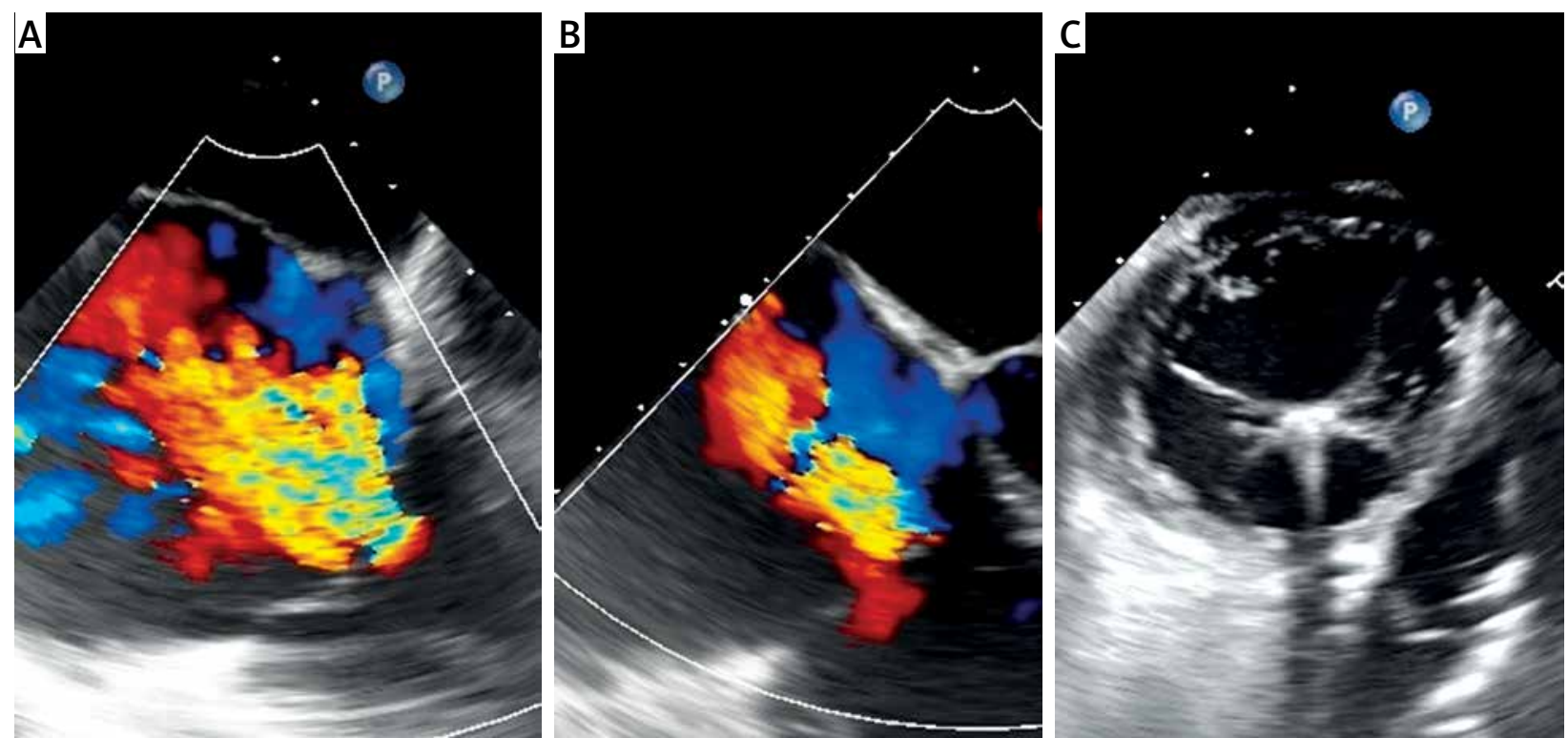

Figure 7. A - Two dimensional transesophageal echocardiography (mid esophageal $0^{\circ}$, 4-chamber view, color Doppler): severe tricuspid regurgitation before tricuspid valve repair using the MitraClip system. B - Two dimensional transesophageal echocardiography (mid esophageal $0^{\circ}, 4$ chamber view, color Doppler): reduction of tricuspid regurgitation (moderate) after tricuspid valve repair using the MitraClip system. $\mathbf{C}-$ Two dimensional transesophageal echocardiography (transgastric $52^{\circ}$, short axis view): double orifice of the tricuspid valve with central clip

tion. The tricuspid valve regurgitation was reassessed after TAVI. The degree of TR was as high as before the TAVI procedure. The degree of mitral valve regurgitation and systolic pulmonary artery pressure did not change after TAVI.
The tricuspid valve edge-to-edge repair using the MitraClip system was performed transfemorally on October, $27^{\text {th }}$ 2015. After puncture of the right femoral vein and after positioning of the steerable guiding catheter in the right atrium, the clip delivery system was introduced into 
the guiding catheter (tip to tip). Then the guiding catheter was angulated slightly in "plus" and "anterior" directions to position the guiding catheter vertical to the tricuspid valve. The next step of the maneuver consisted of advancing the MitraClip device into the right atrium (Figure 5), opening of both arms, navigation (angulation with plus/minus knob; movement with guide handle into anterior direction) and advancing the system into the right ventricle (Figure 6). To be sure to be in the correct perpendicular position, the gastric short axis view (TEE) of the tricuspid valve is necessary, because the rotational movement of the clip is well exposed in this view. By retracting the MitraClip device, both leaflets, anterior and septal, were grasped and closed to coapt the tricuspid leaflets. After an effective reduction of tricuspid regurgitation and achieving a "double orifice" (Figures 7 A-C), the clip was deployed (Figure 8). The clip delivery system and guiding catheter were withdrawn. Finally, the guiding catheter was retracted into the inferior caval vein and explanted. The femoral puncture site was closed using a Z-suture. Reconstruction of the two- and three-dimensional transesophageal echocardiographic dataset documented a significant echocardiographic improvement (Figures 9 A, B): there was an effective reduction of the tricuspid regurgitation (vena contracta area after procedure $0.48 \mathrm{~cm}^{2}$, vena contracta width $5 \mathrm{~mm}$ ). Tricuspid valve stenosis was not detected (mean gradient $1 \mathrm{~mm} \mathrm{Hg}$ ). The patient tolerated both procedures - TA-TAVI and tricuspid valve clipping - very well and was discharged from hospital in an improved health condition on November $\sigma^{\text {th }}, 2015$. The current functional status of the patient 6 months after the procedure is good (NYHA class II-III). The echocardiography showed furthermore a correct position of the tricuspid valve - clip with residual mild tricuspid regurgitation and the ACURATE TA Aortic Bioprosthesis (mean gradient $7 \mathrm{~mm} \mathrm{Hg}$ ) without aortic regurgitation. No mitral regurgitation was observed. The left and right ventricle ejection fraction increased respectively to $38 \%$ and $43 \%$.
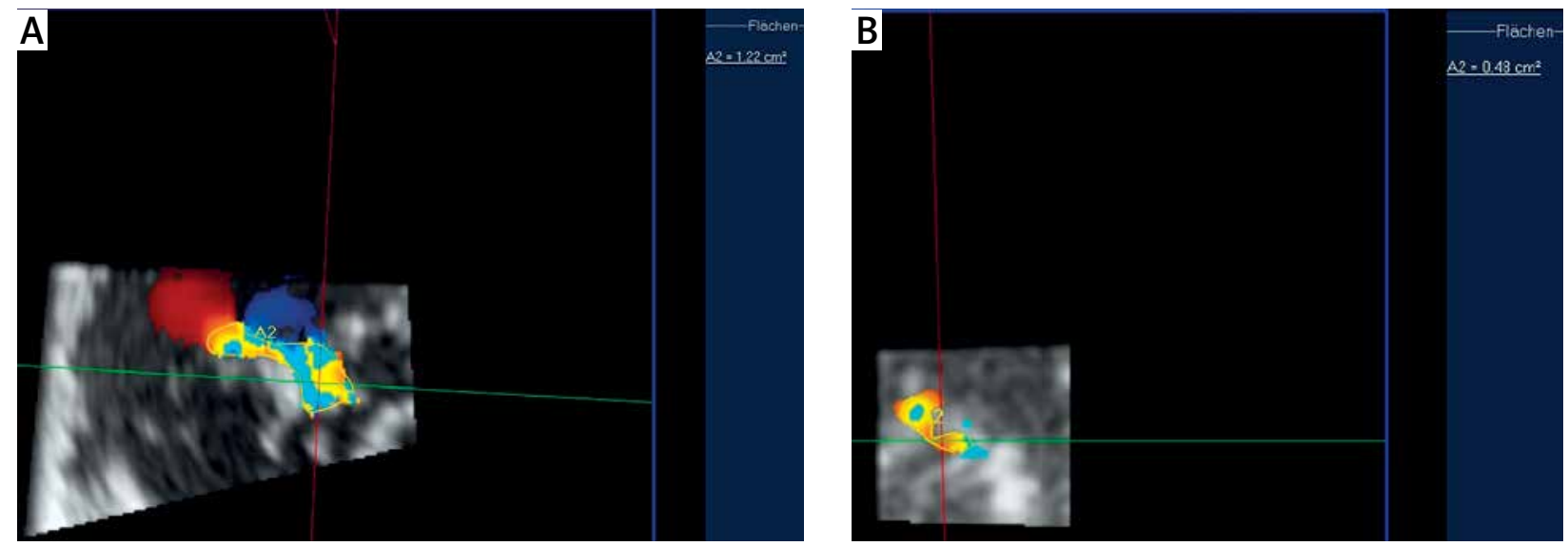

Figure 9. A - Three dimensional transesophageal echocardiography: vena contracta area of tricuspid regurgitation before tricuspid valve repair using the MitraClip system. B - Three dimensional transesophageal echocardiography: vena contracta area of tricuspid regurgitation after tricuspid valve repair using the MitraClip system 
sapical TAVI using the ACURATE TA device followed by transfemoral tricuspid valve edge-to-edge repair using the MitraClip system. Two- and three-dimensional transesophageal echocardiography and fluoroscopy were essential for the precise navigation and placement of the TAVI valve and MitraClip. Elimination of aortic stenosis and a significant reduction of tricuspid regurgitation were associated with decrease of clinical heart failure symptoms. In patients in whom functional tricuspid regurgitation occurred due to dilatation of the RV on the basis of irreversible pulmonary hypertension, exclusive, isolated elimination of the aortic stenosis did not affect the degree of TR. In non-fixed, reversible pulmonary hypertension, after aortic valve replacement a decrease of the right ventricular and annular dilatation and consecutively a reduction of the TR were observed in most cases.

In conclusion, it was found that transapical TAVI and transfemoral tricuspid valve repair using the MitraClip system could be a feasible, safe and well-tolerated therapy for high-risk or inoperable patients, who are non-responders to conservative medical treatment. Future clinical studies are necessary to confirm long-term results of transcatheter edge-to-edge repair of tricuspid regurgitation using the MitraClip system itself and in combination with TAVI in a larger cohort of patients.

\section{Conflict of interest}

Dr. Kowalski has received consultant fee from Abbott for Clinical Advisory Board activity. Dr. Hofmann has received honoraria from Symetis for serving as a proctor. All other authors have no relationships relevant to the contents of this paper to disclose.

\section{References}

1. Feldman T, Kar S, Rinaldi $M$, et al. EVEREST Investigators Percutaneous Mitral Repair With the MitraClip System. Safety and Midterm Durability in the Initial EVEREST (Endovascular Valve Edge-to-Edge REpair STudy) Cohort. J Am Coll Cardiol 2009; 54: 686-94.

2. Feldman T, Foster E, Glower DD, et al.; EVEREST II Investigators. Percutaneous repair or surgery for mitral regurgitation. N Engl J Med 2011; 364: 1395-406.

3. Smith CR, Leon MB, Mack MJ, et al. Transcatheter versus surgical aortic-valve replacement in high-risk patients. N Engl J Med 2011; 364: 2187-98.

4. Leon MB, Smith CR, Mack MJ, et al. Transcatheter aortic-valve implantation for aortic stenosis in patients who cannot undergo surgery. N Engl J Med 2010; 363: 1597-607.

5. Kowalski M, Franz N, Ritter F, et al. Simultaneous transfemoral transcatheter mitral and tricuspid valve edge-to-edge repair (using MitraClip system) completed by atrial septal defect occlusion in a surgically inoperable patient. First-in-human report. Kardiochir Torakochir Pol 2015; 12: 295-7.

6. Schofer J, Tiburtius C, Hammerstingl C, et al. Transfemoral tricuspid valve repair using a percutaneous mitral valve repair system. J Am Coll Cardiol 2016; 67: 889-90.
7. Hammerstingl C, Schueler R, Malasa M, et al. Transcatheter treatment of severe tricuspid regurgitation with the MitraClip system. Eur Heart J 2016; 37: 849-53.

8. Nath J, Foster E, Heidenreich PA, et al. Impact of tricuspid regurgitation on long-term survival. J Am Coll Cardiol 2004; 43: 405-9.

9. Kempfert J, Treede H, Rastan AJ, et al. Transapical aortic valve implantation using a new self-expandable bioprosthesis (ACURATE TA): 6-month outcomes. Eur J Cardiothorac Surg 2013; 43: 52-6. 